\title{
Procesado de datos a través del Láser Escáner Terrestre (TLS): desarrollo de una nueva metodología aplicada a bosques mixtos.
}

\author{
Uzquiano S. ${ }^{* 1,2}$, Bravo F. ${ }^{1,2}$ \\ ${ }^{I}$ Instituto Universitario de Investigación de Gestión Forestal Sostenible. \\ Universidad de Valladolid-INIA. \\ ${ }^{2}$ Dpto. Producción Vegetal y Recursos Forestales. E.T.S. de Ingenierías Agrarias \\ Avenida de Madrid, 57, Palencia (Palencia) 34004.
}

*Autor para correspondencia: sara.uzquiano@alumnos.uva.es

\begin{abstract}
Resumen
Conocer variables dendrométricas para los inventarios forestales de forma rápida y con el mínimo error hoy en día es posible gracias al uso de Láser Escáner Terrestre (TLS) con el que se obtienen nubes de puntos 3D de gran detalle que permite extraer prácticamente cualquier variable del árbol. Sin embargo, el procesado de los datos requiere de mucho tiempo. El objetivo de este estudio ha sido desarrollar una metodología combinando varios programas informáticos (Polyworks, CompuTree y Photoshop) con el fin de agilizar este proceso sobre una masa mixta de Pinus sylvestris y Quercus pyrenaica localizados en norte de España. Se estudiaron 15 árboles de cada especie ajustando modelos de forma básicos de: volumen de copa, diámetro normal, ratio del tronco y altura a la máxima anchura de copa y modelos para evaluar el efecto de la mezcla sobre dichas variables. Los resultados muestran que a través de la metodología seguida se pueden obtener buenas estimaciones para las variables dendrométricas estudiadas. Gracias a este trabajo hemos podido familiarizarnos con nuevos programas utilizados en el proceso de los datos TLS, lo que nos permitirá realizar futuros estudios sobre esta misma masa mixta y profundizar así en el entendimiento de la dinámica de crecimiento de masas mixtas.
\end{abstract}

Palabras clave: Quercus pyrenaica, Pinus sylvestris, modelos de crecimiento, efecto de la mezcla, área basimétrica. 


\section{Introducción}

Obtener información a través de los inventarios forestales es importante para poder evaluar el potencial de nuestros bosques (Moskal and Zhen, 2011, Pretzsch et al., 2016). Por esta razón conseguir acelerar y automatizar el proceso de extracción de estos datos de una forma robusta y precisa se ha convertido en primordial en el ámbito forestal (Piboule et al., 2015).

La estructura del bosque se describe a través de varios atributos asociados con propiedades de masa espacio-temporales como son la altura, la tangencia y cobertura de copas, diámetro a la altura del pecho (DAP) y, volumen de los árboles (Moskal y Zhen, 2011, Latifi et al., 2015). Estos atributos se pueden obtener de forma más o menos sencilla en masas puras, sin embargo en las masas mixtas la obtención de estas variables se complica y es por ello que la dinámica de crecimiento en este tipo de bosques no está aun ampliamente estudiada. Pese a esto, están cobrando mucho interés (Puettmann et al., 2012) ya que son muchos los autores que apuntan hacia una mayor productividad de los bosques mixtos frente a los puros (Río et al., 2016; Morin et al., 2011; Paquette and Messier, 2011) y, otros estudios (BravoOviedo et al., 2014; Kolström et al., 2011) han identificado este tipo de bosques como una adaptación estratégica de cara a la mitigación de los efectos del cambio climático dentro de la gestión de los bosques. Lo que hace necesario tener un mayor conocimiento de cómo funciona la dinámica de estos bosques (Río et al., 2016). Por tanto, cobra mucha importancia el poder conocer las respuestas entre la estructura y el entorno de la masa forestal (Pretzsch et al., 2016). Sin embargo, este hecho rara vez ha sido cuantificado (Pretzsch et al., 2016), ya que la mezcla de especies y sus diversos requerimientos ecológicos complican su modelización en este tipo de masas (Lähde et al., 1999 en Kuuluvainen, 2002).

Con respecto a esta necesidad que se acaba de exponer, la tecnología LiDAR (Light Detection And Ranging) es capaz de proporcionar datos precisos para poder describir la estructura de los bosques debido a su habilidad de captar la estructura tridimensional de los árboles (Latifi, 2012). El estudio de datos LiDAR se han validado sobre varios tipos de masas forestales desde ecosistemas boreales (Hyyppä et al., 2008) hasta tropicales (Drake et al., 2002 and Treuhaft et al., 2010). Dentro de esta tecnología, en los últimos años está tomando mayor protagonismo en el ámbito forestal el dispositivo LiDAR Terrestre (Othmani et al., 2011) al que denominaremos a partir de ahora por sus siglas en inglés TLS (Terrestrial Laser Scanning). Este dispositivo ofrece un gran potencial para el estudio de los bosques proveyendo información espacial y temporal de los mismos (Schneider et al., 2014). Proporciona un gran nivel de detalle, capaz de solventar las limitaciones que presentan los dispositivos convencionales. Es por ello que hoy en día disponemos de un elevado número de estudios en los que se demuestran los atributos de este aparato frente a los métodos tradicionales (Latifi et al., 2015; Bayer et al., 2013; Béland et al., 2011; Côté et al., 2011; Dassot et al., 2012; Seidel et al., 2011 a). Con todo, el reto de este aparato sigue estando en poder acelerar el proceso de extracción de datos para el inventario de una masa 
forestal cuando ésta presenta una elevada densidad de pies por hectárea (Bauwens et al., 2016).

El objetivo del presente estudio es precisamente, implementar una metodología que ayude a acelerar el proceso de extracción de datos y disminuir el sesgo en la obtención de variables dendrométricas y dasométricas desde la nube de puntos generada por TLS a través de la combinación de diferentes software (Polyworks, CompuTree y Photoshop). Para validar el procedimiento propuesto, se ha estimado el Diametro a la Altura del pecho (DAP), Altura total del árbol (HT), Altura a la máxima amplitud de copa (HLCW), altura a la base de la copa (HBC) y el volumen de copa (VC) de 30 árboles de una masa mixta de Pinus sylvestris y Quercus pyrenaica situada en el norte de España.

\section{Material y método}

\section{1 Área de estudio}

La zona de estudio está localizada en el término municipal de Palacio de Valdellorma (León) a $1000 \mathrm{msnm}$, con una temperatura y precipitación media de $9^{\circ} \mathrm{C}$ y $936 \mathrm{~mm}$ respectivamente. El área de estudio tiene una superficie de 2 hectáreas donde se mezcla una masa de plantación de Pinus sylvestris junto con repoblación natural de Quercus pyrenaica y sigue un diseño split-plot dividido en 9 parcelas rectangulares de 50x40m cada (Figura 1) de las cuales, hemos seleccionado tres (A1, A2 y Z2) de las que hemos escaneado y extraído los datos.

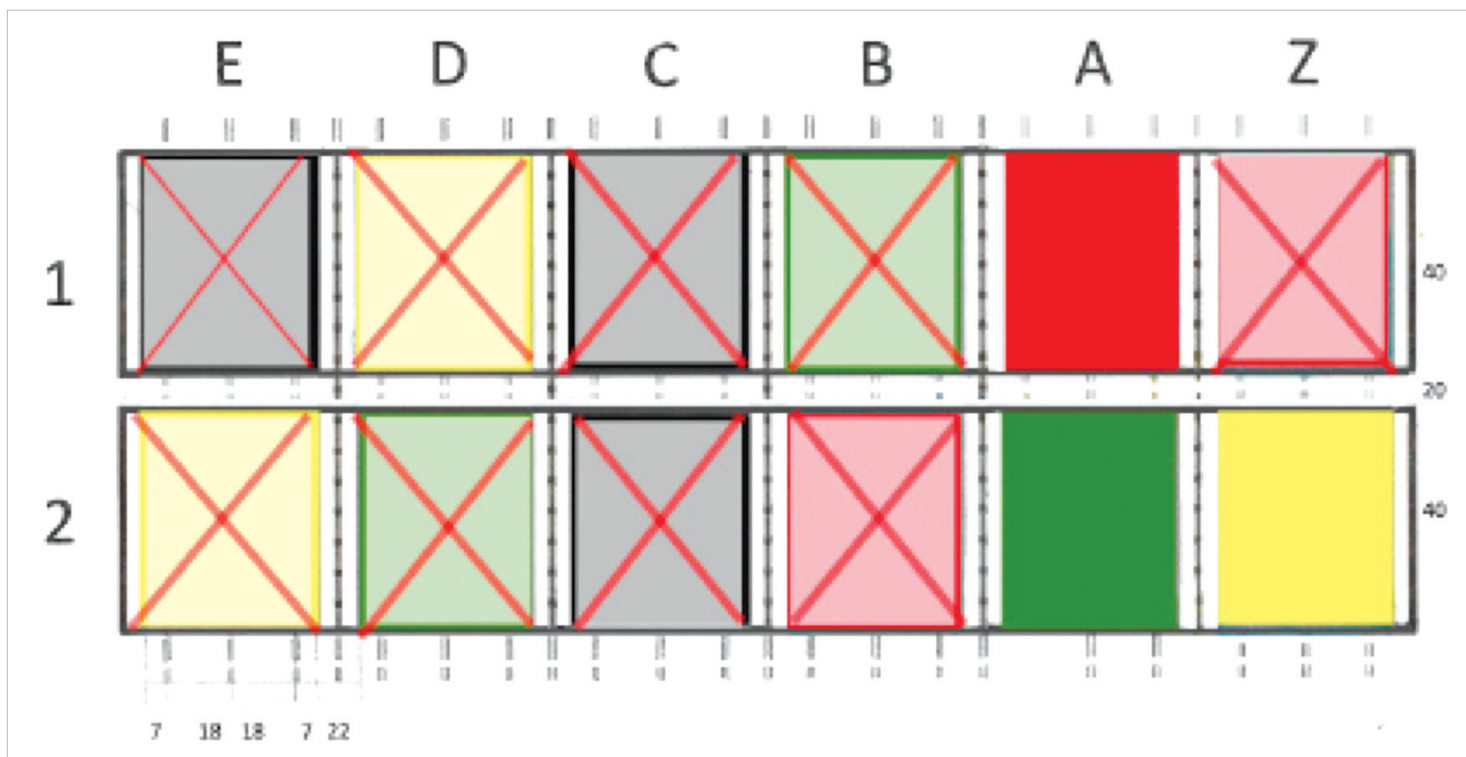

Figura 1. Área de estudio de 2 hectáreas con diseño Split-plot. Las parcelas marcadas en rojo, verde y amarillo son las parcelas A1, A2 y Z2 seleccionadas para el estudio. 


\subsection{Toma de datos}

Los escaneos se llevaron a cabo durante el invierno de 2016 con un escáner TLS Faro Focus 3D. Cada escaneo tuvo una duración de minuto y medio. La totalidad de escaneos tomados varió en función de la densidad de las parcelas. Así en las parcelas A2 y Z2 donde la intensidad de corta es de 0 y del $25 \%$ respectivamente, se tomaron 48 escaneos, mientras que en la parcela A1, que tiene una intensidad de corta del $50 \%$, solamente fueron necesarios 24 escaneos. El tiempo de escaneo de cada parcela fue de 5 horas para las dos primeras parcelas y de 2 horas para la parcela A1.

\subsection{Procesado de datos}

El primer paso es el alineamiento de las tomas, es decir, convertir las imágenes panorámicas (2D) obtenidas por el TLS en nubes de puntos 3D. Este proceso es llevado a cabo de forma automática por el software FaroScene (versión 5.2). A continuación se exportaron los ficheros generados por FaroScene al software Poly-

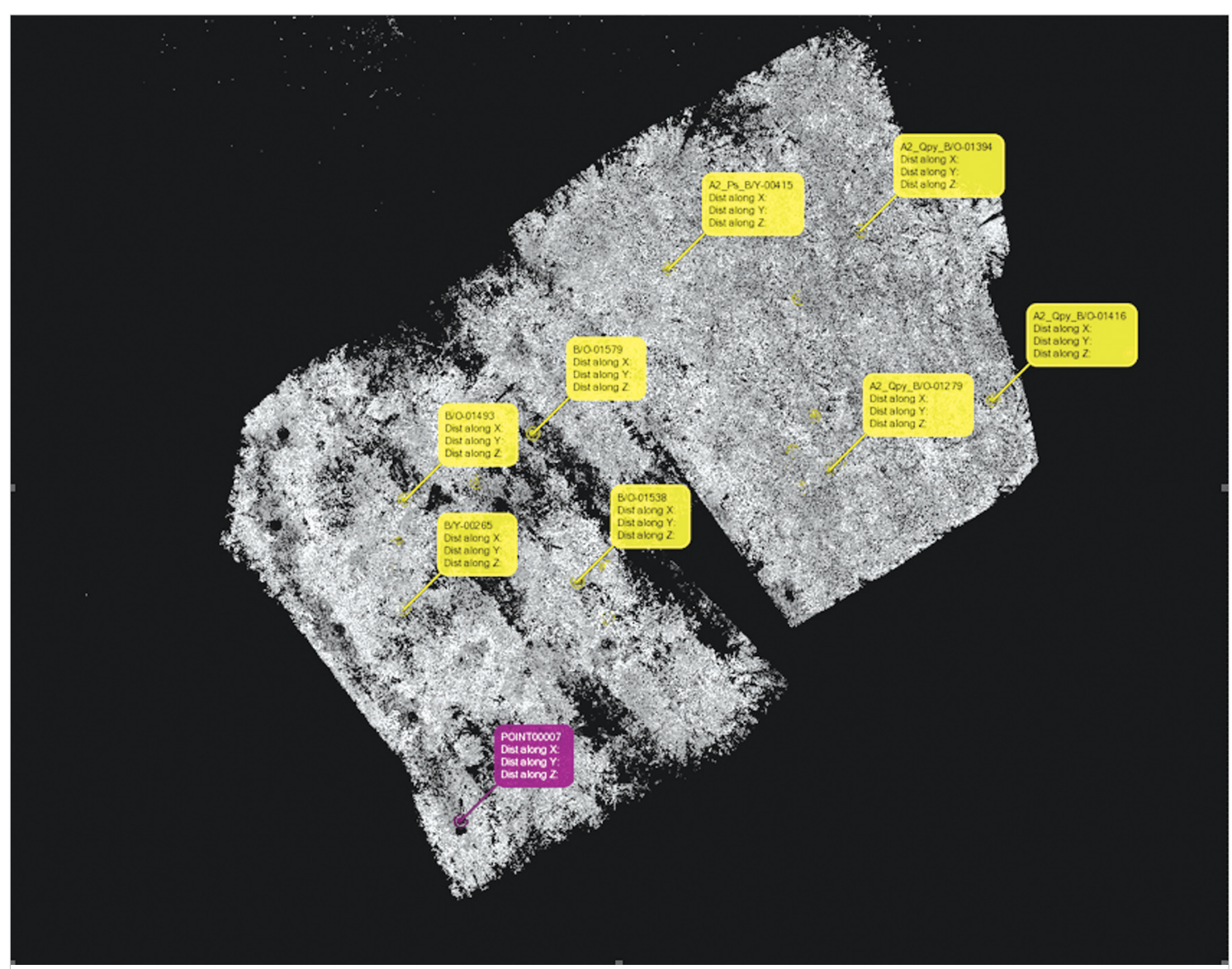

Figura 2. En amarillo las coordenadas de los arboles objeto de estudio tomadas con Estación Total superpuestas sobre las nubes de puntos 3D de las parcelas. 
works versión 12.1.3 (64 bits) concretamente el módulo IM Inspect que permite editar las nubes de puntos.

A continuación identificamos los 30 árboles objeto solapando las coordenadas de dichos árboles (tomadas con una Estación Total en estudios anteriores) con las nubes de puntos generadas por el TLS (Figura 2). Una vez identificados los árboles, el aislamiento de los mismos se ha realizado en el software de uso libre CompuTree (versión 3.4.18) a través de un script desarrollado por el centro de investigación de Nancy (Francia) LerFob - INRA, el cual separa los árboles del suelo e identifica cada pie de forma automática. La parte final del script, que es la selección del árbol se realiza de forma manual. Cada árbol fue exportado en un archivo formato ASCII.

Las variables HT, HBC, HLCW y DAP de cada árbol se obtuvieron volviendo a importar las nubes de puntos en IM Inspect y el volumen del árbol se realizó en CompuTree creando envolventes alrededor del mismo como se muestra en la Figura 3. Finalmente para estudiar el efecto de la mezcla en la alometría de los árboles
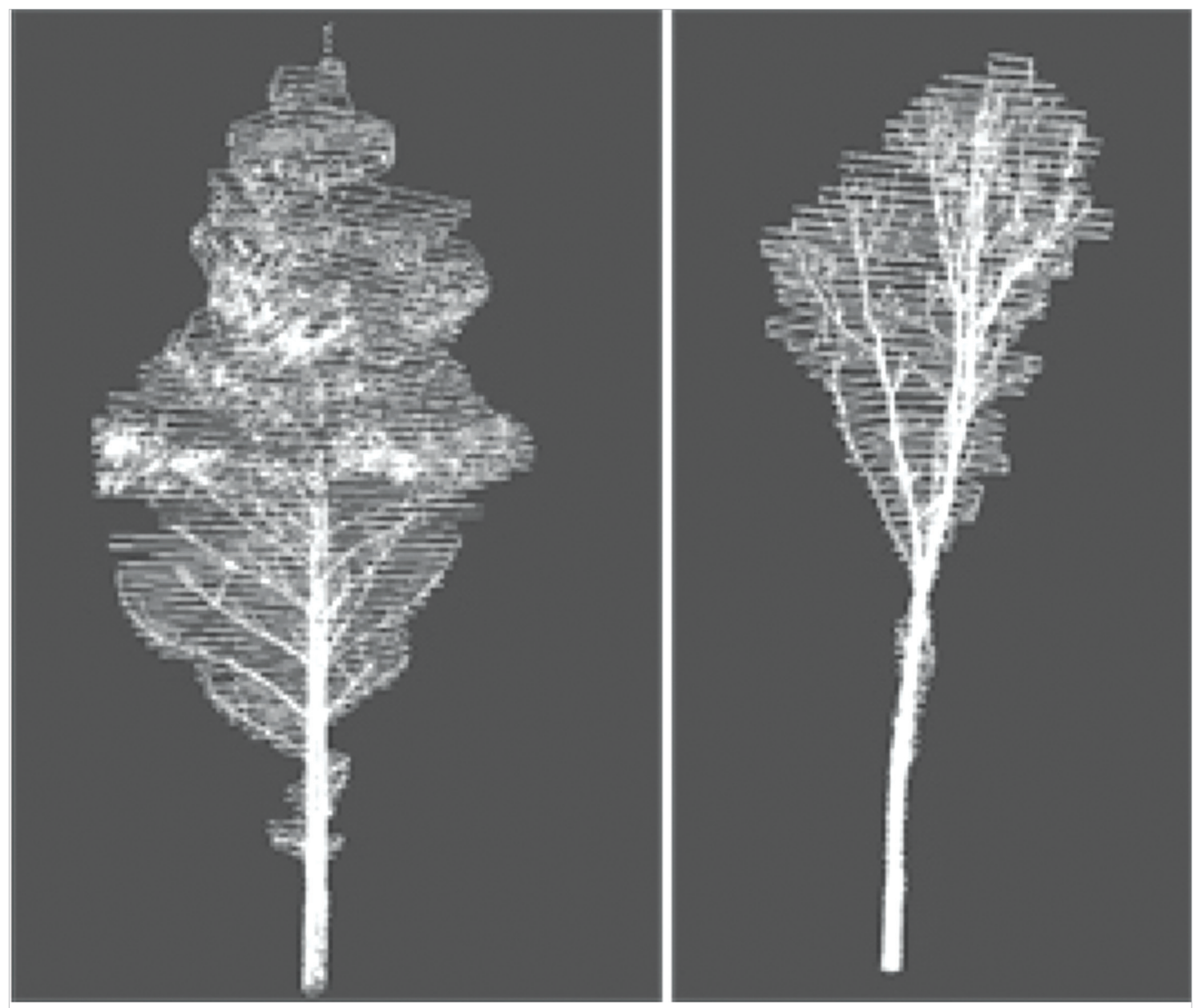

Figura 3. Envolventes creadas alrededor de los árboles cada $10 \mathrm{~cm}$ con el programa CompuTree con el que se ha estudiado el volumen de los árboles. 
se crearon subparcelas circulares de $5 \mathrm{~m}$ de radio (White et al., 2013) alrededor de cada árbol objeto de estudio dentro del programa IM Inspect (Ver Figura 4). Dentro de cada subparcela se midió el DAP de cada árbol y se identificó mezcla entre especies dentro de cada subparcela a través de la proporción de pinos en términos de pie/ha o Área Basimétrica sobre el total de la masa.

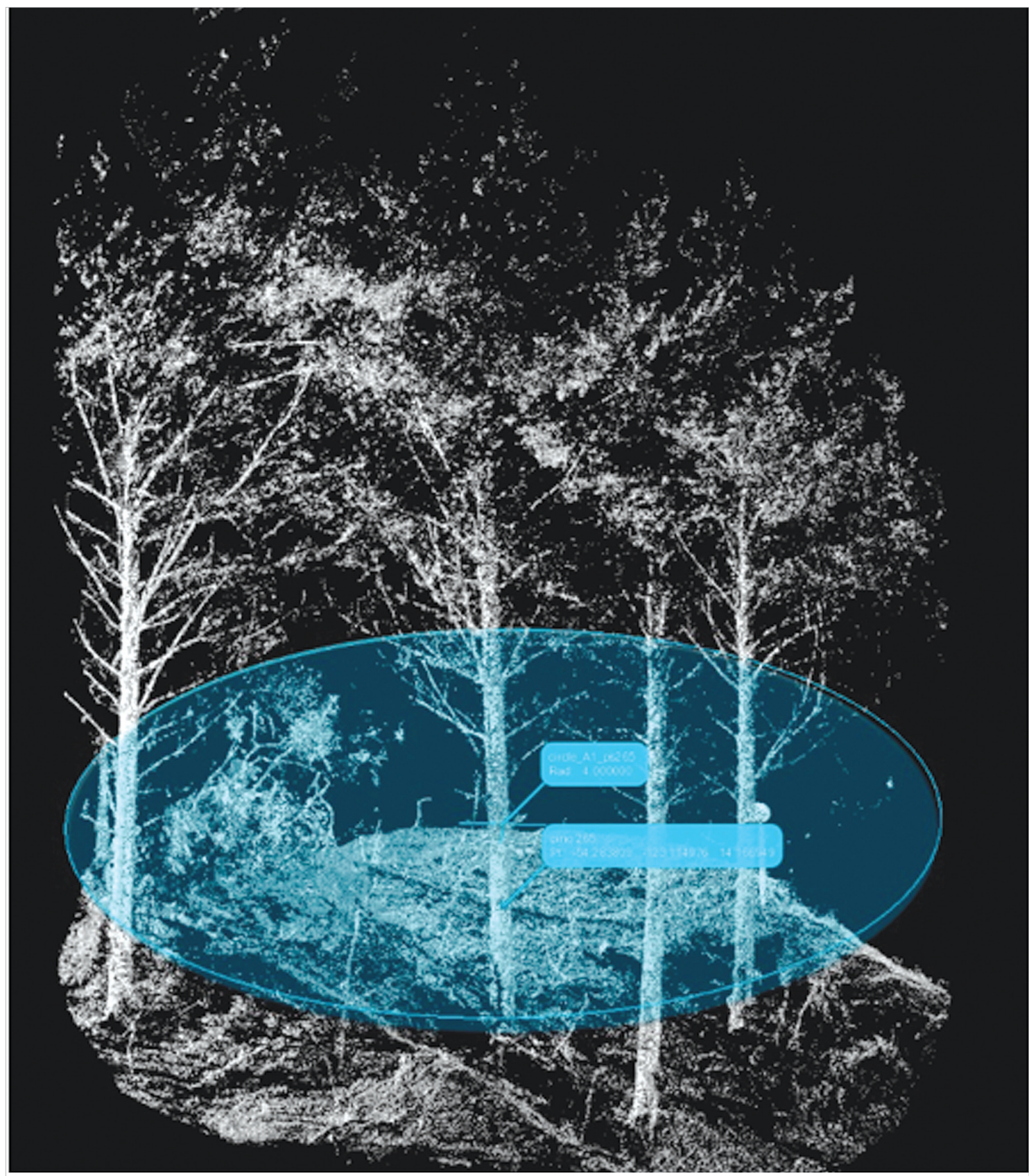

Figura 4. Parcela circular de $5 \mathrm{~m}$ de radio marcado por una circunferencia celeste. En el medio de la imagen marcado el árbol objeto de estudio. 


\subsection{Análisis de datos}

Se utilizaron las siguientes ecuaciones básicas para ajustar los modelos para el VC, HT, HBC y HLCW a través del programa estadístico de libre uso R (R core team, 2016).

- $\log \mathrm{VC}=\mathrm{B} 0+\mathrm{B} 1 * \log (\mathrm{DAP})$

- $\log \mathrm{HT}=\mathrm{B} 0+\mathrm{B} 1 * \log (\mathrm{DAP})+\mathrm{B} 2 * \log (\mathrm{G})$

- $1-(\mathrm{HCB} / \mathrm{HT})=\mathrm{B} 0+\mathrm{B} 1 *(\mathrm{HT} / \mathrm{DAP})$

- $\mathrm{HLCW}=\mathrm{B} 0+\mathrm{B} 1 * \mathrm{HT}+\mathrm{B} 2 * \mathrm{HT}^{2}$

Donde VC = Volumen de copa; DAP = Diámetro a la Altura del Pecho; HT = Altura Total; $\mathrm{G}=$ Diámetro Medio Cuadrático; $\mathrm{HCB}=$ Altura a la Base de la Copa; HLCW = Altura a la Máxima Amplitud de copa. B0, B1 y B2 son los parámetros a ser especificados en cada caso.

Para estudiar el efecto de la mezcla en la alometría de los árboles, sobre las variables significantes obtenidas de los modelos básicos recién expuestos, el parámetro $\mathrm{Bi}$ ha sido remplazado con $\mathrm{Bi} 0+\mathrm{Bi} 1 * \mathrm{MEZCLA}$. Para este estudio, se ha considerado que valores de R2 mayores del $50 \%$ son suficientes para dar el ajuste del modelo por válido.

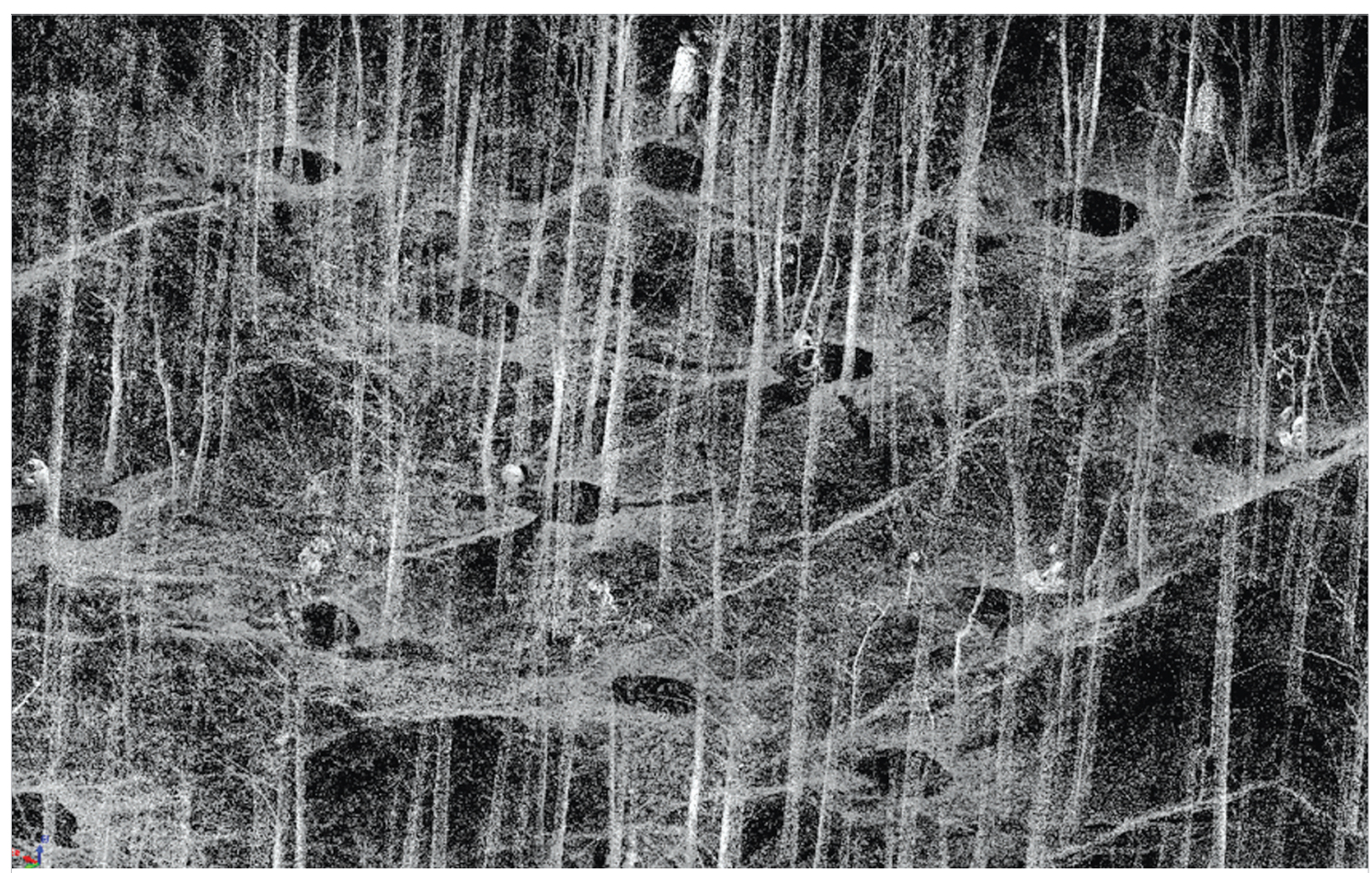

Figura 5. Detalle de la precisión de representación de una de las parcelas de estudio. 


\section{Resultados}

Las nubes de puntos 3D nos brindan una representación exacta de nuestras parcelas tanto de los árboles como del terreno (Figura 5). Además, de proporcionar una georrefenciacion de cada uno de los árboles de la parcela de la misma manera o incluso mejor que las obtenidas con Estación Total, pero en un tiempo mucho menor. La Tabla 1 muestra de forma resumida los resultados de la estadística descriptiva de las variables dendrométricas para cada especie y de la misma manera la Tabla 2 muestra la estadística descriptiva para las variables dasométricas para cada parcela y especie.

Tabla 1. Estadística descriptiva de las variables nedidas a través de las nubes de puntos 3D. La tabla muestra en las filas: número de pies (n), media $(\overline{\mathrm{x}})$ máximo (Max), mínimo (min) y desviación típica $(\sigma)$. En las columnas: $\mathrm{HC}$ es la altura de copa. $\mathrm{V}_{\text {tronco }}$ es el volumen del tronco, $\mathrm{V}_{\text {copa }}$ es el volumen de copa y $\mathrm{V}_{\text {total }}$ es el volumen total del árbol.

\begin{tabular}{|c|c|c|c|c|c|c|c|c|}
\hline & $\begin{array}{l}\text { DBH } \\
\text { (cm) }\end{array}$ & $\begin{array}{c}\mathbf{H t} \\
(\mathrm{m})\end{array}$ & $\begin{array}{c}\text { HBC } \\
(\mathrm{m})\end{array}$ & $\begin{array}{l}\mathrm{HC} \\
(\mathrm{m})\end{array}$ & $\underset{\text { (m) }}{\text { HLCW }}$ & $\begin{array}{l}V_{\text {tronco }} \\
\left(\mathbf{m}^{3}\right)\end{array}$ & $\begin{array}{l}V_{\text {copa }} \\
\left(\mathbf{m}^{3}\right)\end{array}$ & $\begin{array}{l}V_{\text {total }} \\
\left(\mathbf{m}^{3}\right)\end{array}$ \\
\hline \multicolumn{9}{|c|}{ Pinus sylvestris } \\
\hline $\mathrm{n}$ & 15 & 15 & 15 & 15 & 15 & 15 & 15 & 15 \\
\hline$\overline{\mathrm{x}}$ & 16,78 & 10,51 & 4,13 & 6,39 & 6,88 & 0,22 & 26,42 & 26,60 \\
\hline Max. & 21,38 & 12,50 & 5,80 & 9,40 & 8,70 & 0,57 & 64,30 & 64,46 \\
\hline Min. & 9,38 & 7,30 & 2,00 & 4,30 & 3,10 & 0,11 & 2,33 & 2,39 \\
\hline$\sigma$ & 3,41 & 1,48 & 1,07 & 1,28 & 1,75 & 0,14 & 18,25 & 18,29 \\
\hline \multicolumn{9}{|c|}{ Quercus pyrenaica } \\
\hline $\mathrm{n}$ & 15 & 15 & 15 & 15 & 15 & 15 & 15 & 15 \\
\hline$\overline{\mathrm{x}}$ & 11,88 & 9,02 & 5,17 & 3,85 & 7,45 & 0,35 & 4,95 & 5,30 \\
\hline Max. & 17,93 & 12,00 & 7,30 & 5,90 & 9,90 & 1,90 & 19,19 & 19,64 \\
\hline Min. & 7,10 & 5,90 & 2,70 & 2,50 & 3,40 & 0,04 & 0,34 & 0,38 \\
\hline$\sigma$ & 2,82 & 1,51 & 1,21 & 1,08 & 1,61 & 0,54 & 4,73 & 4,96 \\
\hline
\end{tabular}

Al estudiar los modelos de Volumen de copa, altura total, fracción de copa y altura a la máxima amplitud de copa para cada una de las especies, se obtuvieron resultados significativos para los modelos de forma de las dos especies excepto para la fracción de copa de Quercus pyrenaica con lo que se eliminó del estudio. Las ecuaciones resultantes se muestran en la Tabla 3. Con estos resultados se estudió como se ven dichas variables afectadas por la proporción de especies (Tabla 4) aunque en este caso no se obtuvo ningún resultado significativo por lo que, con los datos analizados, no hemos detectado que la mezcla de especies afecte a los modelos propuestos para las 3 variables dendrométricas. 
Tabla 2. Estadística descriptiva de las variables nedidas a través de las nubes de puntos 3D. La tabla muestra en las filas: el número de pies estudiados $(\mathrm{n})$, la media $(\overline{\mathrm{x}})$ máximo (Max), mínimo (min) y desviación típica $(\sigma)$. En las columnas: las variables dasométricas. Área Basimétrica $(\mathrm{AB})$, número de pies por hectárea (N), Área Basimétrica de las subparcelas (G), Diámetro medio cuadrado (Dg) e Índice de Reineke (S).

\begin{tabular}{|c|c|c|c|c|c|}
\hline & $\begin{array}{c}\mathrm{AB} \\
\left(\mathrm{m}^{2}\right)\end{array}$ & $\underset{\left(\text { pies }^{*} h^{-1}\right)}{\mathbf{N}}$ & $\underset{\left(\mathbf{m}^{2^{*}} \mathbf{h a}^{-1}\right)}{\mathbf{G}}$ & Dg & $\mathbf{S}$ \\
\hline \multicolumn{6}{|c|}{ Pinus sylvestris } \\
\hline $\mathrm{n}$ & 263 & 263 & 263 & 263 & 263 \\
\hline$\overline{\mathrm{x}}$ & 24,16 & 3143,73 & 56,38 & 35,42 & 888,62 \\
\hline Max. & 263,00 & 5092,96 & 263,00 & 263,00 & \#\#\#\#\# \\
\hline Min. & 0,21 & 263,00 & 26,81 & 10,06 & 263,00 \\
\hline$\sigma$ & 79,21 & 1473,71 & 68,80 & 75,51 & 274,72 \\
\hline \multicolumn{6}{|c|}{ Quercus pyrenaica } \\
\hline $\mathrm{n}$ & 313 & 313 & 313 & 313 & 313 \\
\hline$\overline{\mathrm{x}}$ & 39,30 & 1692,77 & 60,96 & 48,90 & 600,76 \\
\hline Max. & 313,00 & 2546,48 & 313,00 & 313,00 & 895,77 \\
\hline Min. & 0,07 & 313,00 & 8,90 & 2,28 & 226,47 \\
\hline$\sigma$ & 110,59 & 876,64 & 102,34 & 106,78 & 275,88 \\
\hline
\end{tabular}

Tabla 3. Ecuaciones testadas para cada especie.

\begin{tabular}{|c|c|c|}
\hline Modelo & Especie & Ecuación \\
\hline \multirow{2}{*}{ Volumen de Copa } & P. sylvestris & $\log C V=\beta 0+\beta 1 * \log (D B H)$ \\
\cline { 2 - 3 } & Q. Pyrenaica & $\log H t=\beta 0+\beta 1 * \log (D B H)+\beta 2 * \log (G)$ \\
\hline \multirow{2}{*}{ Altura Total } & P. sylvestris & $\log H t=\beta 0+\beta 1 * D B H^{2}+\log (G)$ \\
\cline { 2 - 3 } & Q. Pyrenaica & $1-\left(\frac{\overline{H B C}}{H T}\right)=\beta 0+\beta 1\left(\frac{H t}{D A P}\right)$ \\
\hline \multirow{3}{*}{ Fracción de Copa } & P. sylvestris & -- \\
\cline { 2 - 3 } & Q. Pyrenaica & $H L C W=\beta 0+\beta 1+H t+\sqrt{H t}$ \\
\hline \multirow{2}{*}{ HLCW } & P. sylvestris & $H L C W=\beta 0+\beta 1+H t+\beta 2 * H t^{2}$ \\
\cline { 2 - 3 } & Q. Pyrenaica & \\
\hline
\end{tabular}

Tabla 3. Ecuaciones testadas para cada especie.

\begin{tabular}{|c|c|c|}
\hline Modelo & Especie & Ecuación \\
\hline \multirow{2}{*}{ Volumen de Copa } & P. sylvestris & $\log C V=\beta 00 * M I X+\beta 01 * \log (D B H) * M I X$ \\
\cline { 2 - 3 } & Q. Pyrenaica & $\log H t=\beta 00 * M I X+\beta 01 *(\log (D B H) * M I X+\beta 02 * \log (G))$ \\
\hline \multirow{2}{*}{ Altura Total } & P. sylvestris & $\log H t=\beta 00 * M I X+\left(\beta 01 * D B H^{2} * M I X\right)+\log (G)$ \\
\cline { 2 - 3 } & Q. Pyrenaica & $H L C W=\beta 00+\beta 01+H t * M I X+\sqrt{H t}$ \\
\hline \multirow{2}{*}{ HLCW } & P. sylvestris & $H L C W=\beta 0+\beta 1+H t * M I X+\beta 2 * H t^{2}$ \\
\cline { 2 - 3 } & Q. Pyrenaica & \\
\hline
\end{tabular}




\section{Discusión}

La velocidad y la objetividad en la recolección de datos son atributos deseados en la inventariación de masas forestales que el TLS es capaz de proporcionarnos (Hopkinson, 2004) sin embargo el procesado de los datos captados con este aparato requiere de mucho tiempo (Uzquiano et al., 2014; Xu et al., 2013). La introducción del software CompuTree, comparado con otro estudio realizado previamente (Uzquiano, 2014) ha reducido el tiempo de procesamiento de los datos, pero aún queda mucho camino por recorrer.

En la realización de este estudi, al igual que Dassot et al. (2012), el tiempo de escaneo en campo es dependiente del terreno y de la cantidad de pies/ha que haya en la parcela. Siendo las 3 parcelas de estudio del mismo tamaño, en la A2 y Z2 donde la densidad de pies/ha es bastante elevada se tardó una media de 5 horas en escanear cada una de las parcelas. Sin embargo en la parcela A1 cuya densidad de parcela ha sido cortada al 50\% se tardó una media de 2 horas en obtener escaneada la totalidad de la parcela. Si bien, el TLS en el ámbito forestal no es aún operacional al mismo nivel que lo es el LiDAR Aéreo (ALS) (Hauglin et al., 2014). Éste es capaz de proporcionarnos información con un gran nivel de detalle como es el posicionamiento exacto de los árboles o su DAP, impensables de obtener con los métodos convencionales.

Respecto al procesado de nubes de puntos, la utilización de CompuTree es una buena herramienta para aislar árboles automáticamente sin sesgo, pero el inconveniente principal de este programa es que no puede procesar datos mayores de $44 \mathrm{mi}-$ llones de nubes de puntos, es decir, no mayores de $2 \mathrm{~Gb}$ (Othmani et al., 2011) por
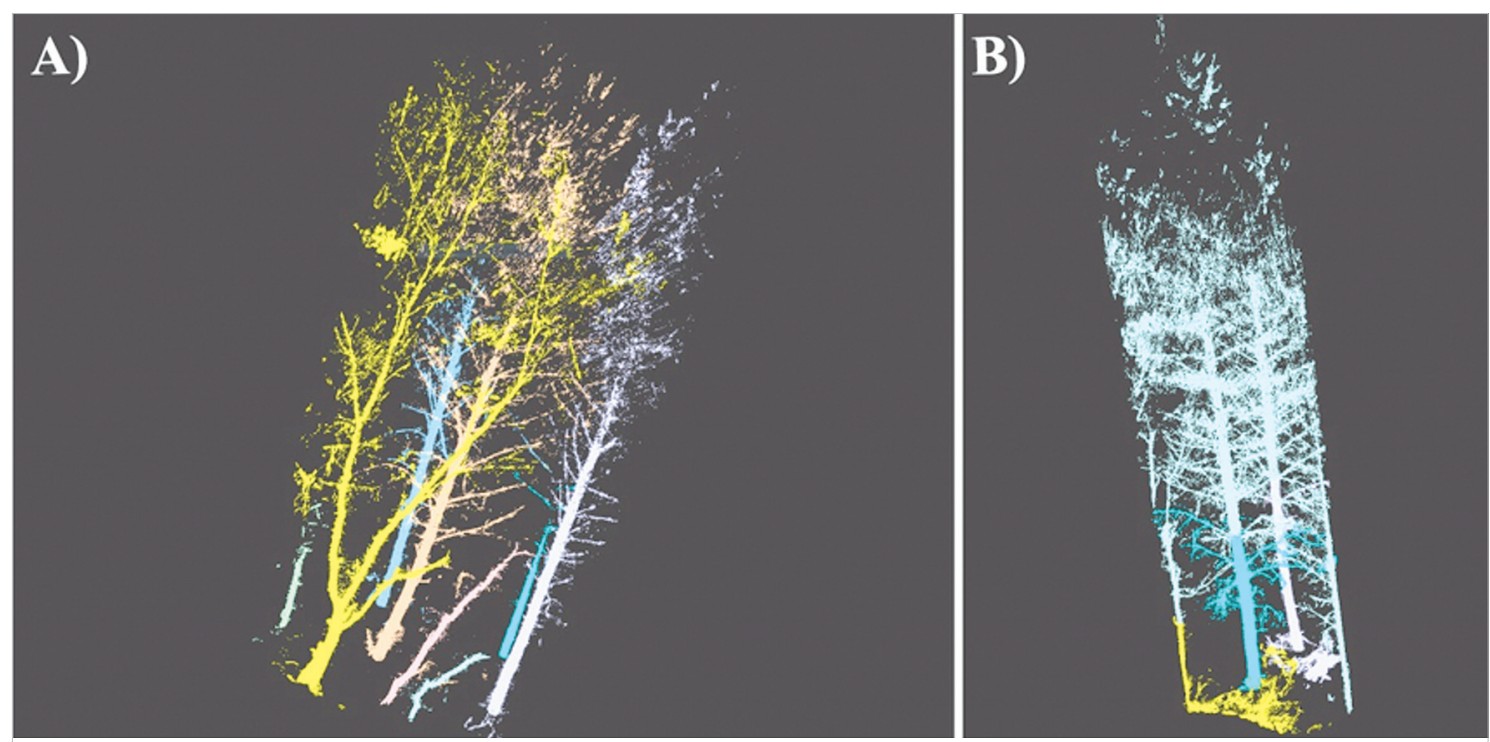

Figura 6. El programa CompuTree identifica cada árbol mediante colores. Figura A) muestra el buen reconocimiento de pies en la parcela A1 donde la densidad era baja. La figura B) muestra un problema de identificación de pies en la parcela de control A2 donde la densidad es elevada. 
lo que toca dividir cada una de las parcelas pues cada una ocupa alrededor de 20 Gb. A pesar de eso, el aislamiento de árboles fue rápido y preciso (Figura 6.A) para la mayoría de los casos. Solo en la parcela de control A2, donde la densidad de pies/ha era la más elevada, y las copas se encontraban entrelazadas, el programa identificó todas las copas juntas como parte de un solo árbol (Figura 6.B). El proceso de aislamiento del árbol es el más lento pues el programa identifica cada árbol, pero solo se puede aislar y exportar un sólo árbol a la vez, pero una vez aislado podemos obtener mucha información para cada árbol como queda demostrado en este estudio (Volumen, DAP, Árbol alto, etc.) en solo unos minutos y minimizar los errores y sesgos en los mismos. Otra de las grandes ventajas que da este programa es su capacidad para exportar los datos en numerosos formatos.

Respecto al ajuste de modelos, encontramos que, al igual que en otros estudios (Dassot et al., 2012, Hauglin et al., 2014, Raumonen et al., 2015), el, a priori, escaso número de muestras analizadas (15 para cada especie) es suficiente para validar los ajustes realizados en los modelos de forma estudiados a partir de datos TLS. Sin embargo, no es así para la validación de los modelos teniendo en cuenta la mezcla entre especies. Esto puede ser debido a varios factores:

1.- Bajo número de observaciones.

2.- La proporción de especies se midió a través del área basimétrica ya que es la forma más común de definir la proporción de especies (Légaré et al., 2004; Perot y Picard, 2012; Groot et al., 2014 en Bravo-Oviedo et al., 2014).

\section{Conclusión}

Al igual que en nuestro estudio previo (Uzquiano et al., 2014) y en la línea de e.g. Raumonen et al., 2015; Dassot et al., 2012 la automatización en el procesado de los datos TLS aún no puede ser aplicado a gran escala. Sin embargo los resultados obtenidos demuestran que la metodología aplicada en este trabajo para la extracción de datos nos proporciona unos datos más robustos y precisos impensables de conseguir con los métodos convencionales. Los datos TLS nos proporcionan un importante respaldo para poder hacer un estudio de la evolución de esta masa forestal y su estructura dentro de unos años.

\section{Bibliografía}

Bauwens, S., Bartholomeus, H., Calders, K., \& Lejeune, P. (2016). Forest Inventory with Terrestrial LiDAR: A Comparison of Static and Hand-Held Mobile Laser Scanning. Forests, 7(6), 127. http://doi.org/10.3390/f7060127

Bayer, D., Seifert, S., Pretzsch, H., (2013). Structural crown properties of Norway spruce (Picea abies [L.] Karst.) and European beech (Fagus sylvatica [L.]) in mixed versus pure stands revealed by terrestrial laser scanning. Trees 27, 1035-1047. http://dx.doi. org/10.1007/s00468-013-0854-4 
Béland, M., Widlowski, J.L., Fournier, R.A., Côté, J.F., Verstraete, M.M., (2011). Estimating leaf area distribution in savanna trees from terrestrial LiDAR measurements. Agric. For. Meteorol. 151, 1252-1266.

Bravo-Oviedo, A., Pretzsch, H., Ammer, C., Andenmatten, E., Barbati, A., Barreiro, S., \& Ouden, J. D. (2014). European Mixed Forests: definition and research perspectives. Forest systems, 23(3), 518-533.

Côté, J.F., Fournier, R.A., Egli, R., (2011). An architectural model of trees to estimate forest structural attributes using terrestrial LiDAR. Environ. Model. Softw. 26, 761-777.

Dassot, M., Colin, A., Santenoise, P., Fournier, M., \& Constant, T. (2012). Terrestrial laser scanning for measuring the solid wood volume, including branches, of adult standing trees in the forest environment. Computers and Electronics in Agriculture, 89, 86-93.

Drake, J.B., Dubayah, R.O., Knox, R.G., Clark, D.B., Blair, J.B., (2002). Sensitivity of largefootprint LiDAR to canopy structure and biomass in a neotropical rainforest. Remote Sens. Environ. 81 (2-3), 378-392

Groot A, Adhikary S, Sharma M, Luckai N, Bell FW, Larocque GR, (2014). Effect of species composition on the production rate and efficiency of young Picea glaucaPopulus tremuloides forests. Forest Ecology and Management 315: 1-11. http://dx.doi. org/10.1016/j.foreco.2013.12.017

Hyyppä, J., Hyyppä, H., Leckie, D., Gougeon, F., Yu, X., Maltamo, M., (2008). Review of methods of small-footprint airborne laser scanning for extracting forest inventory data in boreal forests. Int. J. Remote Sens. 29 (5), 1339-1366.

Hauglin, M., Gobakken, T., Astrup, R., Ene, L., \& Næsset, E. (2014). Estimating SingleTree Crown Biomass of Norway Spruce by Airborne Laser Scanning: A Comparison of Methods with and without the Use of Terrestrial Laser Scanning to Obtain the Ground Reference Data. Forests, 5(3), 384-403. http://doi.org/10.3390/f5030384

Hopkinson, C., Chasmer, L., Young-Pow, C., Treitz, P., (2004). Assessing Forest Metrics with a Ground-Based Scanning Lidar. Canadian Journal of Forest Research. vol. 34, no. 3, pp. 573-583.

Kolström M, Lindner M, Vilén T, Maroschek M, Seidl R, Lexer MJ, Netherer S, Kremer A, Delzon S, Barbati A, Marchetti M, Corona P, (2011). Reviewing the science and implementation of climate change adaptation measures in European forestry. Forests 2: 961-982.

Kuuluvainen, T. (2002). Introduction: disturbance dynamics in boreal forests: defining the ecological basis of restoration and management of biodiversity. SilvaFennica, 36(1), 512.

Latifi, H., Nothdurft, A., Straub, C., Koch, B., (2012). Modelling stratified forest attributes using optical/LiDAR features in a central European landscape. Int. J. Digit. Earth 5 (2), $106-132$

Latifi, H., Fassnacht, F. E., Müller, J., Tharani, A., Dech, S., \& Heurich, M. (2015). Forest inventories by LiDAR data: A comparison of single tree segmentation and metric-based methods for inventories of a heterogeneous temperate forest. International Journal of AppliedEarthObservation and Geoinformation, 42, 162-174.

Légaré S, Paré D, Bergeron Y. (2004). The responses of black spruce growth to an increased proportion of aspen in mixed stands. Canadian Journal of Forest Research 34: 405-416. http://dx.doi.org/10.1139/x03-251

Morin, X., Fahse, L., Scherer-Lorenzen, M., Bugmann, H., (2011). Tree species richness 
promotes productivity in temperate forests through strong complementarity between species: species richness promotes forest productivity. Ecol. Lett. 14, 1211-1219. http:// dx.doi.org/10.1111/j.14610248.2011.01691.x

Moskal, L.M.,and Zheng, G. (2011). Retrieving Forest Inventory Variables with Terrestrial Laser Scanning (TLS) in Urban Heterogeneous Forest. Remote Sensing, 4(12), 1-20. http://doi.org/10.3390/rs4010001

Othmani, A., Piboule, A., Krebs, M., Stolz, C., Lew Yan Voon, L., \& Lew Yan Voon Towards, L. (2011). Towards automated and operational forest inventories with T-Lidar.

Paquette, A., Messier, C., (2011). The effect of biodiversity on tree productivity: from temperate to boreal forests. Glob. Ecol. Biogeogr. 20, 170-180. http://dx.doi.org/ 10.1111/j.1466-8238.2010.00592.x

Perot T, Picard N. (2012). Mixture enhances productivity in a two-species forest: evidence from a modeling approach. Ecological Research 27: 83-94. http://dx.doi.org/10.1007/ s11284-011-0873-9

Piboule, A., Krebs, M., Esclatine, L., \&Hervé, J.C. (2015). Computree: a collaborativeplat formfor use of terrestrial LiDAR in dendrometry.

Pretzsch, H., del Río, M., Schütze, G., Ammer, C., Annighöfer, P., Avdagic, A., BravoOviedo, A. (2016). Mixing of Scots pine (Pinus sylvestris L.) and European beech (Fagus sylvatica L.) enhances structural heterogeneity, and the effect increases with water availability. Forest Ecology and Management, 373, 149-166. http://doi.org/ 10.1016/j.foreco.2016.04.043

Puettmann, K.I., Coates, K.D., Messier, C.C. (2012). A Critique of Silviculture: Managing for Complexity. Island Press.

Raumonen, P., Casella, E., Calders, K., Murphy, S., Akerblom, M., \& Kaasalainen, M. (2015). massive-scale tree modelling from TLS data. http://doi.org/10.5194/ isprsannals-II-3-W4-189-2015

Río, M., Pretzsch, H., Alberdi, I., Bielak, K., Bravo, F., Brunner, A., BravoOviedo, A. (2016). Characterization of the structure, dynamics, and productivity of mixed-species stands: review and perspectives. European Journal of Forest Research. http://doi.org/ 10.1007/s10342-015-0927-6

Schneider, F.D., Leiterer, R., Morsdorf, F., Gastellu-Etchegorry, J.P., Lauret, N., Pfeifer, N., \& Schaepman, M.E. (2014). Simulating imaging spectrometer data: 3D forest modeling based on LiDAR and in situ data. RemoteSensing of Environment, 152, 235-250.

Seidel, D., Leuschner, C., Müller, A., Krause, B., (2011a). Crown plasticity in mixed forests - quantifying asymmetry as a measure of competition using terrestrial laser scanning. For. Ecol. Manage. 261, 2123-2132.

Treuhaft, R.N., Goncalves, F.G., Drake, J.B., Chapman, B.D., dos Santos, J.R., Dutra, L.V., Graca, P.M.L.A., Purcell, G.H., (2010). Biomass estimation in a tropical wet forest using Fourier transforms of profiles from LiDAR or interferometric SAR. Geophys. Res. Lett. 37, L23403.

Uzquiano,S. (2014). Mediciones dendrométricas y dasométricas mediante técnicas LiDAR y fotogramétricas. Proyecto Fin de Máster de Ingeniería de Montes. Escuela Técnica Superior de Ingenierías Agrarias de Palencia. Universidad de Valladolid. http://uva doc.uva.es/handle/10324/6587

Uzquiano, S., Martínez, J., San Martín, R., Bravo, F. (2014). Mediciones dendrométricas y dasométricas mediante técnicas LiDAR y fotogramétricas. Nota técnica Cuad. Soc. 
Esp. Cienc. For. 40: 193-202. http://secforestales.org/publicaciones/index.php/cuader nossecf/index

White, J.C., Wulder, M.A., Varhola, A., Vastaranta, M., Coops, N.C., Cook, B.D., Pitt, D., Woods, M. (2013). A best practices guide for generating forest inventory attributes from airborne laser scanning data using an area-based approach (Version 2.0). Natural Resources Canada. Canadian Forest Service. Canadian Wood Fibre Centre. Information Report FI-X-010.

Xu, W., Su, Z., Feng, Z., Xu, H., Jiao, Y., Yan, F. (2013). Comparison of conventional measurement and LiDAR based measurement for crown structures. Computers and Electronics in Agriculture, vol. 98, pp. 242-251. 